\title{
On Minty's theorem in the Heisenberg group
}

\author{
A. Calogero* and R. Pini*+
}

July 2, 2018

\begin{abstract}
In this paper we extend some classical results of Convex Analysis to the sub-Riemannian setting of the Heisenberg group. In particular, we provide a horizontal version of Minty's theorem concerning maximal $\mathrm{H}$-monotone operators defined in the Heisenberg group with values in the first layer of its Lie algebra.
\end{abstract}

Key words: Heisenberg group, convexity, subdifferential, monotone map, cyclic monotone map, maximal monotonicity

MSC: Primary: 26B25 Secondary: 47H05; 53C17

\section{Introduction}

Maximal monotone maps in Euclidean spaces and, more in general, in Hilbert spaces, play key roles in several settings, specifically in fixed point theorems and in solving generalized equations. A well known result, the celebrated Minty theorem, provides a characterization of maximal monotonicity (see [13]): given a monotone set-valued map $T: X \rightrightarrows X$, where $X$ is a Hilbert space, then $T$ is maximal monotone if and only if $I+\lambda T$ is surjective onto $X$, for every $\lambda>0$; in this case, the map $(I+\lambda T)^{-1}$ is single-valued on $X$. A remarkable implication of Minty's theorem is the possibility of approximating $T$ in some sense by singlevalued maps $T_{\lambda}$, called Yosida approximations, that are also maximal monotone and are defined as $T_{\lambda}=\left(I-J_{\lambda}\right) / \lambda$, where $J_{\lambda}=(I+\lambda T)^{-1}$.

The most notable example of a maximal monotone map arises as the subdifferential $\partial f$ of a convex function $f: \mathbb{R}^{n} \rightarrow \mathbb{R}$. More precisely, the subdifferential of the function $f$ at $x$ is defined as the following subset of $\mathbb{R}^{n}$ :

$$
\partial f(x):=\left\{v \in \mathbb{R}^{n}: f(y) \geq f(x)+\langle v, y-x\rangle, \quad \forall y \in \mathbb{R}^{n}\right\} ;
$$

the subdifferential map is therefore given by:

$$
\partial f: \mathbb{R}^{n} \rightrightarrows \mathbb{R}^{n}, \quad x \mapsto \partial f(x) .
$$

In this case, if $T=\partial f$, then the classical Moreau theorem provides a useful approximation of $f$ via convex and Fréchet differentiable functions $f_{\lambda}$, converging upward to $f$, and whose gradient is given by the Yosida approximation $(\partial f)_{\lambda}$.

\footnotetext{
*Dipartimento di Matematica e Applicazioni, Università degli Studi di Milano-Bicocca, Via Cozzi 53, 20125 Milano, Italy (andrea.calogero@unimib.it, ${ }^{+}$corresponding author: rita.pini@unimib.it)
} 
The aim of this paper is to start the investigation of a possible extension of these results, that are peculiar of Convex Analysis, to the sub-Riemannian background, starting from the simplest situation of the Heisenberg group $(\mathbb{H}, \circ)$. In this framework, a first step towards this study makes use of the concept of convexity and subdifferentiability of a function $u: \mathbb{H} \rightarrow \mathbb{R}$. In the last few years several notions of convexity have been introduced in the Heisenberg group and, more generally, in Carnot groups, but the notion of horizontal convexity (H-convexity) turned out to be the most suitable to match their sub-Riemannian structure (see [9], [10]). The naturally associated H-subdifferential of a function $u$ at a point $g \in \mathbb{H}$ is the (possibly empty) subset of the first layer $V_{1}$ of the Lie algebra of $\mathbb{H}$ :

$$
\partial_{H} u(g):=\left\{p \in V_{1}: u(g \circ \exp w) \geq u(g)+\langle w, p\rangle, \forall w \in V_{1}\right\},
$$

where $\circ$ denotes the group law in $\mathbb{H}$, and $V_{1}$ can be identified with $\mathbb{R}^{2}$ (see Section 2 for the details). The H-subdifferential map is therefore the set-valued map

$$
\partial_{H} u: \mathbb{H} \rightrightarrows V_{1}, \quad g \mapsto \partial_{H} u(g) .
$$

It is worthwhile noticing that, while in the Euclidean case the subdifferential at a point can be identified with a subset of the same space $X^{*}=X$, in the framework of the Heisenberg group there is a reduction in dimension passing from $I H$ to $V_{1}$; this fact will give rise to some pathological effects.

The notion of H-monotonicity, that turns out to be a particular case of the more general notion of $c$ H-monotonicity (see [7]), fits the monotonicity of maps in Euclidean spaces to the horizontal structure of $\mathbb{H}$. As in the Euclidean case, the notion of H-convexity for functions is strictly related to the notion of $\mathrm{H}$-monotonicity of the H-subdifferential map. In Section 3 we show that the map $\partial_{H} u$, if $u: \mathbb{H} \rightarrow \mathbb{R}$ is H-convex, is maximal H-monotone.

More generally, we consider a map $T: \mathbb{H} \rightrightarrows V_{1}$, which is maximal monotone in the horizontal sense and try to see whether a suitable version of Minty's type theorem still holds. In Section 4, we prove our main result, that is, a horizontal version of Minty's theorem in a slightly weaker form:

Theorem 1.1 Let $T: \mathbb{H} \rightrightarrows V_{1}$ be an $H$-monotone operator with $\operatorname{dom}(T)=\mathbb{H}$. If $T$ is maximal H-cyclically monotone, then the map $\left.\left(\xi_{1}+\lambda T\right)\right|_{H_{g}}$ is surjective onto $V_{1}$ for every $g \in \mathbb{H}$.

Conversely, if the map $\left.\left(\xi_{1}+\lambda T\right)\right|_{H_{g}}$ is surjective onto $V_{1}$ for every $g \in \mathbb{H}$, then $T$ is maximal H-monotone.

Here $H_{g}$ denotes the horizontal plane associated to the point $g \in \mathbb{H}$, i.e. the set of all the points in $\mathbb{H}$ that are reachable from $g$ via horizontal segments (see Section 2 for the details). Nevertheless, despite one is lead to foresee a possible parallelism with the Euclidean case, this one fails when trying to show that, for a fixed $g \in \mathbb{H}$, the map $\left(\xi_{1}+\lambda T\right)^{-1}: V_{1} \rightrightarrows H_{g}$ is single-valued. As a matter of fact, we show (see Example 4.1) that this is no longer true even in the case $T=\partial_{H} u$, where $u$ is an $\mathrm{H}$-convex function on $\mathbb{H}$. This fact is quite unexpected, for, in this case, there is no change in dimension when we pass from $V_{1}$ to the horizontal plane $H_{g}$, whose topological dimension is 2 .

\section{Preliminaries}

The Heisenberg group $\mathbb{H}$ is the Lie group given by the underlying manifold $\mathbb{R}^{3}$ with the non commutative group law

$$
g \circ g^{\prime}=(x, y, t) \circ\left(x^{\prime}, y^{\prime}, t^{\prime}\right)=\left(x+x^{\prime}, y+y^{\prime}, t+t^{\prime}+2\left(x^{\prime} y-x y^{\prime}\right)\right),
$$


unit element $e=(0,0,0)$, and $g^{-1}=(-x,-y,-t)$. Left translations and anisotropic dilations are, in this setup, $L_{g_{0}}(g)=g_{0} \circ g$ and $\delta_{\lambda}(x, y, t)=\left(\lambda x, \lambda y, \lambda^{2} t\right)$.

The differentiable structure on $\mathbb{H}$ is determined by the left invariant vector fields

$$
X=\partial_{x}+2 y \partial_{t}, \quad Y=\partial_{y}-2 x \partial_{t}, \quad T=\partial_{t}, \quad \text { with }[X, Y]=-4 T .
$$

The vector field $T$ commutes with the vector fields $X$ and $Y ; X$ and $Y$ are called horizontal vector fields.

The Lie algebra $\mathfrak{h}$ of $\mathbb{H}$ is the stratified algebra $\mathfrak{h}=\mathbb{R}^{3}=V_{1} \oplus V_{2}$, where $V_{1}=$ $\operatorname{span}\{X, Y\}, V_{2}=\operatorname{span}\{T\} ;\langle\cdot, \cdot\rangle$ will denote the inner product. Via the exponential map $\exp : \mathfrak{h} \rightarrow \mathbb{H}$ we identify the vector $\alpha X+\beta Y+\gamma T$ in $\mathfrak{h}$ with the point $(\alpha, \beta, \gamma)$ in $\mathbb{H}$; the inverse $\xi: \mathbb{H} \rightarrow \mathfrak{h}$ of the exponential map has the unique decomposition $\xi=\left(\xi_{1}, \xi_{2}\right)$, with $\xi_{i}: \mathbb{H} \rightarrow V_{i}$. Since we identify $V_{1}$ with $\mathbb{R}^{2}$ when needed, $\xi_{1}: \mathbb{H} \rightarrow V_{1} \sim \mathbb{R}^{2}$ is given by $\xi_{1}(x, y, t)=(x, y)$. We say that $\gamma:[0,1] \rightarrow \mathbb{H}$ is a horizontal segment if $\gamma(\lambda)=g \circ \delta_{\lambda}(\exp w)=g \circ \exp (\lambda w)$ for some $g \in \mathbb{H}$ and $w \in V_{1}$ fixed. For more details on the structure of the Heisenberg group see, e.g., [8] and [5].

The main issue in the analysis on the Heisenberg group is that the classical differential operators are considered only in terms of the horizontal fields. For any open subset $\Omega$ of $\mathbb{H}$, let us denote by $\Gamma^{1}(\Omega)$ the class of functions having continuous derivatives with respect to the vector fields $X$ and $Y$. We recall that the horizontal gradient of a function $u \in \Gamma^{1}(\Omega)$ at $g \in \Omega$ is the 2 -vector

$$
\left(\nabla_{H} u\right)(g)=((X u)(g),(Y u)(g)),
$$

written with respect to the basis $\{X, Y\}$ of $V_{1}$.

The notion of horizontal subdifferential of a function $u: \mathbb{H} \rightarrow \mathbb{R}$ at a point $g$ takes into account the sub-Riemannian structure of $\mathbb{H}$. This horizontal structure relies on the notion of horizontal plane: given a point $g_{0} \in \mathbb{H}$, the horizontal plane $H_{g_{0}}$ associated to $g_{0}$ is the plane in $\mathbb{H}$ defined by

$$
\begin{aligned}
H_{g_{0}}=L_{g_{0}}\left(\exp \left(V_{1}\right)\right) & =\left\{g=(x, y, t): t=t_{0}+2 y_{0} x-2 x_{0} y\right\} \\
& =\left\{g_{0} \circ \exp w, w \in V_{1}\right\} .
\end{aligned}
$$

We note that $g^{\prime} \in H_{g}$ if and only if $g \in H_{g^{\prime}}$.

Definition 2.1 Let $u: \Omega \rightarrow \mathbb{R}$, where $\Omega$ is a subset of $\mathbb{H}$. The horizontal subdifferential (or $H$-subdifferential) of $u$ at $g \in \Omega$ is the set

$$
\partial_{H} u(g)=\left\{v \in V_{1}: u(g \circ \exp w) \geq u(g)+\langle v, w\rangle, \quad \forall w \in V_{1}: g \circ \exp w \in \Omega\right\} .
$$

If $v \in \partial_{H} u(g)$, we say that $v$ is an $H$-subgradient of $u$ at $g$. The set-valued map

$$
\partial_{H} u: \mathbb{H} \rightrightarrows V_{1}, \quad g \mapsto \partial_{H} u(g)
$$

is called the H-subdifferential of $u$.

Let us recall that, given a set-valued map $T: \mathbb{H} \rightrightarrows V_{1}$, its domain is defined as $\operatorname{dom}(T):=\{g \in \mathbb{H}: T(g) \neq \emptyset\}$, and its graph is the subset of $\mathbb{H} \times V_{1}$ given by $\operatorname{gph}(T):=\{(g, v): g \in \operatorname{dom}(T), v \in T(g)\}$.

Convex functions in the Heisenberg group setting were first introduced by Luis Caffarelli (in unpublished work from 1996). This notion did not really surface in the literature until 2002, when it was independently formulated and studied, in the more general setting of Carnot groups, in [10] and in [9]. Essentially, a convex function in $\mathbb{H}$ is a function whose restriction to horizontal segments are Euclidean convex functions of one variable. 
Definition 2.2 (H-convexity) A function $u: \Omega \subset \mathbb{H} \rightarrow \mathbb{R}$ is called $H$-convex if

$$
u(g \circ \exp (\lambda v)) \leq u(g)+\lambda(u(g \circ \exp v)-u(g))
$$

for every $g \in \Omega, v \in V_{1}$ and $\lambda \in[0,1]$, provided $g \circ \exp (\lambda v) \in \Omega$.

Despite the notion of H-convexity requires a suitable behavior only on the horizontal segments, H-convex functions enjoy some nice regularity properties, as local Lipschitz continuity (see [3]) and hence differentiability almost everywhere in horizontal directions.

Likewise the Euclidean case, the relation between H-convexity and H-subdifferentiability of a function is not unexpected. The investigation of this connection started in the pioneering work [9], and it was carried out in [6]: we mention that in the recent paper [4] the authors study this connection without involving any group structure. In our context, the following result holds:

Theorem 2.1 (see Proposition 10.5 in [9] and Theorem 4.4 in [6]) Let $\Omega$ be an open subset of $\mathbb{H}$, and $u: \Omega \rightarrow \mathbb{R}$. Then $u$ is $H$-convex if and only if $\partial_{H} u(g) \neq \emptyset$, for every $g \in \Omega$.

It is worthwhile noticing that if $u$ is H-convex and $u \in \Gamma^{1}(\Omega)$, then $\partial_{H} u(g)=\left\{\nabla_{H} u(g)\right\}$ for every $g \in \Omega$.

\section{On the H-subdifferential map of an $\mathrm{H}$-convex function}

The main result of this section, Theorem 3.2, concerns the relationship between H-convex functions and the monotonicity properties of their subdifferential maps.

The notions of H-convexity for a function $u: \mathbb{H} \rightarrow \mathbb{R}$, and of the $\mathrm{H}$-subdifferential map $\partial_{H} u: \mathbb{H} \rightrightarrows V_{1} \sim \mathbb{R}^{2}$ given in the previous section, supply the guidelines in order to introduce and to study the notion of monotonicity for set-valued maps $T: \mathbb{H} \rightrightarrows V_{1}$.

First of all, consider an H-convex function $u: \mathbb{H} \rightarrow \mathbb{R}$. For every $g \in \mathbb{H}$ and $g^{\prime} \in H_{g}$ we obtain, by (1),

$$
u(g) \geq u\left(g^{\prime}\right)+\left\langle v, \xi_{1}\left(g^{\prime}\right)-\xi_{1}(g)\right\rangle
$$

for every $v \in \partial_{H} u(g)$. Since $g \in H_{g^{\prime}}$, on the other hands we obtain

$$
u\left(g^{\prime}\right) \geq u(g)+\left\langle v^{\prime}, \xi_{1}(g)-\xi_{1}\left(g^{\prime}\right)\right\rangle,
$$

for every $v^{\prime} \in \partial_{H} u\left(g^{\prime}\right)$. Keeping the comparison between Euclidean notions and horizontal ones, we provide the following

Definition 3.1 (H-monotonicity) Given a set-valued map $T: \mathbb{H} \rightrightarrows V_{1}, T$ is said to be H-monotone if

$$
\left\langle v^{\prime}-v, \xi_{1}\left(g^{\prime}\right)-\xi_{1}(g)\right\rangle \geq 0,
$$

for every $g \in \operatorname{dom}(T), g^{\prime} \in \operatorname{dom}(T) \cap H_{g}, v \in T(g), v^{\prime} \in T\left(g^{\prime}\right)$.

In order to introduce the notion of H-cyclic monotonicity, we say that the set $\left\{g_{i}\right\}_{i=0}^{n} \subset$ $\mathbb{H}, n>0$, is an $H$-sequence if $g_{i+1} \in H_{g_{i}}$, for every $i=0, \ldots, n-1$. An H-sequence is closed if $g_{n} \in H_{g_{0}}$; in this case, we usually set $g_{n+1}=g_{0}$. In particular, if we consider an H-convex function as before, and a closed H-sequence $\left\{g_{i}\right\}_{i=0}^{n}$, we obtain

$$
u\left(g_{i+1}\right) \geq u\left(g_{i}\right)+\left\langle v_{i}, \xi_{1}\left(g_{i+1}\right)-\xi_{1}\left(g_{i}\right)\right\rangle,
$$

for every $v_{i} \in \partial_{H} u\left(g_{i}\right), i=1, \ldots, n$. As in the Euclidean framework, adding up the two sides of all the previous $n$ inequalities we are lead to the following notion (see Definition 6.1 in $[7])$ : 
Definition 3.2 (H-cyclic monotonicity) We say that $\mathcal{R} \subset \mathbb{H} \times V_{1}$ is an H-cyclically monotone set if, for every sequence $\left\{\left(g_{i}, v_{i}\right)\right\}_{i=0}^{n} \subset \mathcal{R}$ such that $\left\{g_{i}\right\}_{i=0}^{n}$ is a closed H-sequence, we have that

$$
\sum_{i=0}^{n}\left\langle\xi_{1}\left(g_{i+1}\right), v_{i}\right\rangle \leq \sum_{i=0}^{n}\left\langle\xi_{1}\left(g_{i}\right), v_{i}\right\rangle
$$

A set-valued map $T: \mathbb{H} \rightrightarrows V_{1}$ is an $H$-cyclically monotone map if $\operatorname{gph}(T)$ is $H$-cyclically monotone.

It is clear that, for an H-convex function $u$, the inequalities (2) and (3) entail that $\partial_{H} u$ : $I H \rightrightarrows V_{1}$ is H-monotone; moreover, summing up the $n$ inequalities in (4), we get that $\partial_{H} u$ is $\mathrm{H}$-cyclically monotone. In the following we will provide an example of an H-monotone map that is not H-cyclically monotone (see Example 3.1).

A first study of the properties of H-monotonicity appears in [6] where the authors prove (see Theorem 6.4) that if $u: \mathbb{H} \rightarrow \mathbb{R}$ is H-convex, then

$$
u(g)=u\left(g_{0}\right)+\sup _{\mathcal{P}}\left\{\sum_{i=0}^{n-1}\left\langle v_{i}, \xi_{1}\left(g_{i+1}\right)-\xi_{1}\left(g_{i}\right)\right\rangle\right\},
$$

where $g_{0} \in H$ is fixed and

$$
\mathcal{P}=\left\{\left\{\left(g_{i}, v_{i}\right)\right\}_{i=0}^{n} \subset \operatorname{gph}\left(\partial_{h} u\right),\left\{g_{i}\right\}_{i=0}^{n} \text { closed H-sequence, } n>0\right\} ;
$$

the right hand side of (6) is called Rockafellar function. In the proof of this result the $\mathrm{H}$-cyclic monotonicity of $\operatorname{gph}\left(\partial_{H} u\right)$ is crucial. The role of the horizontal version of the Rockafellar function and the connection between H-cyclically monotone sets and H-convex functions is emphasized in a subsequent result in [7]. A useful version of Theorem 6.6 in [7] in our context is the following:

Theorem 3.1 Let $T: \mathbb{H} \rightrightarrows V_{1}$ be an $H$-cyclically monotone map with $\operatorname{dom}(T)=\mathbb{H}$. Then there exists an $H$-convex function $u: \mathbb{H} \rightarrow \mathbb{R}$ such that

$$
\operatorname{gph}(T) \subset \operatorname{gph}\left(\partial_{H} u\right)
$$

We emphasize that the function $u$ mentioned in the previous result is a Rockafellar function.

It is well known that in the Euclidean case the notion of maximality is crucial. We say that the set-valued map $T: \mathbb{H} \rightrightarrows V_{1}$ is maximal $H$-monotone (maximal $H$-cyclically monotone) if there are no H-monotone (H-cyclically monotone) set-valued maps $T^{\prime}: \mathbb{H} \rightrightarrows$ $V_{1}$ such that $T(g) \subset T^{\prime}(g)$ for every $g \in \mathbb{H}$, and $T\left(g^{\prime}\right) \subsetneq T^{\prime}\left(g^{\prime}\right)$, for some $g^{\prime} \in \mathbb{H}$.

Notice that any H-cyclically monotone map that is maximal H-monotone, is maximal H-cyclically monotone, i.e. there are no H-cyclically monotone maps $T^{\prime}$ such that $\operatorname{gph}(T) \subsetneq$ $\operatorname{gph}\left(T^{\prime}\right)$.

In the following the next remark will be of some use:

Remark 3.1 Let $T: \mathbb{H} \rightrightarrows V_{1}$ be H-monotone. Then $T$ is maximal H-monotone if and only if for all $(g, v) \notin \operatorname{gph}(T)$, there exists $g^{\prime} \in H_{g}$ and $v^{\prime} \in T\left(g^{\prime}\right)$ such that

$$
\left\langle v-v^{\prime}, \xi_{1}(g)-\xi_{1}\left(g^{\prime}\right)\right\rangle<0
$$

The main result of this section is the following 


\section{Theorem 3.2}

i. If $u: \mathbb{H} \rightarrow \mathbb{R}$ is an $H$-convex function, then the set-valued map $T=\partial_{H} u: \mathbb{H} \rightrightarrows V_{1}$ is maximal $H$-monotone; since it is $H$-cyclically monotone, it is also maximal H-cyclically monotone.

ii. If $T: \mathbb{H} \rightrightarrows V_{1}$ is a maximal H-cyclically monotone with $\operatorname{dom}(T)=\mathbb{H}$, then there exists an $H$-convex function $u$ such that $T=\partial_{H} u$.

Proof: i. Let $u: \mathbb{H} \rightarrow \mathbb{R}$ be H-convex. First of all notice that, from Theorem 4.4 in [6], $\operatorname{dom}(T)=\mathbb{H}$. We have just seen at the beginning of this section that the monotonicity of $\partial_{H} u$ follows trivially from the H-convexity of $u$. Straightforward computations show that $\partial_{H} u$ is also H-cyclically monotone, according to Definition 3.2.

Let us prove the maximal monotonicity. Fix $g \in \mathbb{H}$, and take any $v \notin \partial_{H} u(g)$; by the definition of horizontal subgradient, there exists $g^{\prime} \in H_{g}, g^{\prime}=g \circ \exp z$ for some $z \in V_{1}$, such that

$$
u\left(g^{\prime}\right)<u(g)+\left\langle v, \xi_{1}\left(g^{\prime}\right)-\xi_{1}(g)\right\rangle .
$$

Set $\phi(\cdot)=u(\cdot)-\left\langle v, \xi_{1}(\cdot)-\xi_{1}(g)\right\rangle$; we have that

$$
\phi\left(g^{\prime}\right)<\phi(g) .
$$

From the H-convexity of $\phi: \mathbb{H} \rightarrow \mathbb{R}$, it is clear that the restriction of $\phi$ to the horizontal segment $\left[g, g^{\prime}\right] \subset H_{g} \cap H_{g^{\prime}}$, i.e. the function $\lambda \rightarrow \phi(g \circ \exp (\lambda z)), \lambda \in[0,1]$, is Euclidean convex. Let us notice that

$$
\phi\left(g \circ \exp (\lambda z) \circ \exp \left(\lambda^{\prime} z\right)\right)=\phi\left(g \circ \exp \left(\left(\lambda+\lambda^{\prime}\right) z\right)\right) .
$$

Then, from (9), there exists $\tilde{\lambda} \in(0,1)$ such that, for $\tilde{g}=g \circ \exp (\tilde{\lambda} z) \in H_{g}$,

$$
\lim _{\lambda \rightarrow 0^{+}} \frac{\phi(\tilde{g} \circ \exp (\lambda z))-\phi(\tilde{g})}{\lambda}<0
$$

Let us denote by $\phi^{\prime}(\tilde{g} ; z)$ the previous limit. Now take any $\tilde{v} \in \partial_{H} u(\tilde{g})$. Then Proposition 4.1 in [6] implies that

$$
u^{\prime}(\tilde{g} ; v) \geq\langle\tilde{v}, v\rangle, \quad \forall v \in V_{1}
$$

in particular, $u^{\prime}(\tilde{g} ; z) \geq\langle\tilde{v}, z\rangle$. Moreover, from the definition of $\phi$,

$$
u^{\prime}(\tilde{g} ; z)=\phi^{\prime}(\tilde{g} ; z)+\langle v, z\rangle<\langle v, z\rangle,
$$

and therefore, recalling that $\tilde{\lambda}>0$,

$$
\tilde{\lambda}\langle\tilde{v}-v, z\rangle=\left\langle\tilde{v}-v, \xi_{1}(\tilde{g})-\xi_{1}(g)\right\rangle<0,
$$

contradicting the H-monotonicity of $\partial_{H} u$.

ii. Suppose now that $T$ is maximal H-cyclically monotone with $\operatorname{dom}(T)=\mathbb{H}$. Then, Theorem 3.1 implies that there exists an H-convex function $u$ such that $T(g) \subset \partial_{H} u(g)$, for every $g \in \mathbb{H}$. From the maximal H-cyclic monotonicity of $T$ and i., we get that $T=\partial_{H} u$.

As in [1], Example 12.7, the following result holds in the Heisenberg setting: 
Proposition 3.1 Let $T: \mathbb{H} \rightrightarrows V_{1}$ be a continuous $H$-monotone and single-valued map with $\operatorname{dom}(T)=\mathbb{H}$. Then $T$ is maximal H-monotone.

Proof: Let $g \in \mathbb{H}$ and $v \in V_{1}$ such that

$$
\left\langle v-T\left(g^{\prime}\right), \xi_{1}(g)-\xi_{1}\left(g^{\prime}\right)\right\rangle \geq 0, \quad \forall g^{\prime} \in H_{g} ;
$$

we will prove that $v=T(g)$. Set $g^{\prime}=g \circ \exp \left(-\lambda\left(\xi_{1}(\tilde{g})-\xi_{1}(g)\right)\right)$, for some $\tilde{g} \in \mathbb{H}$ and $\lambda>0$; we obtain, from the previous inequality and dividing by $\lambda$,

$$
\left\langle v-T\left(g \circ \exp \left(-\lambda\left(\xi_{1}(\tilde{g})-\xi_{1}(g)\right)\right)\right), \xi_{1}(\tilde{g})-\xi_{1}(g)\right\rangle \geq 0, \quad \forall \lambda>0 .
$$

Taking the limit as $\lambda \rightarrow 0^{+}$, we obtain

$$
\left\langle v-T(g), \xi_{1}(\tilde{g})-\xi_{1}(g)\right\rangle \geq 0
$$

the generality of $\tilde{g}$ implies that $v=T(g)$.

Remark 3.2 Let $\tilde{T}: \mathbb{R}^{2} \rightrightarrows \mathbb{R}^{2}$ and $T: \mathbb{H} \rightrightarrows V_{1}=\mathbb{R}^{2}$ be maps with $\operatorname{dom}(\tilde{T})=\mathbb{R}^{2}$, $\operatorname{dom}(T)=$ IH, such that

$$
T(x)=\tilde{T}\left(\xi_{1}(x)\right), \quad \forall x \in \mathbb{H} .
$$

If $\tilde{T}$ is cyclically monotone, then $T$ is H-cyclically monotone. The converse is false, in general.

This difference between the "Euclidean" cyclic monotonicity of $T$ and the H-cyclic monotonicity of $\tilde{T}$ is delicate, as the next example shows.

Example 3.1 Let us consider $\tilde{T}: \mathbb{R}^{2} \rightarrow \mathbb{R}^{2}$ defined by $\tilde{T}(x, y)=Q(x, y)^{T}$, where $Q$ is a $2 \times 2$ matrix. It is well-known (see [12], p. 240) that $\tilde{T}$ is monotone if and only if $\frac{1}{2}\left(Q+Q^{T}\right)$ is positive semidefinite; moreover $\tilde{T}$ is cyclically monotone if and only if $Q$ is symmetric and positive semidefinite.

Let us consider the particular case $Q=\left(\begin{array}{cc}3 & 2 \\ -2 & 4\end{array}\right)$ : in this case, $\tilde{T}$ is maximal monotone (note that $\tilde{T}$ is continuous). Define the map $T: \mathbb{I} \rightrightarrows V_{1}=\mathbb{R}^{2}$ as in (10): explicitly,

$$
T(x, y, t)=\tilde{T}(x, y)=(3 x+2 y,-2 x+4 y) .
$$

Consider now the function $u: \mathbb{H} \rightarrow \mathbb{R}$ defined by

$$
u(x, y, t)=\frac{3}{2} x^{2}+2 y^{2}+t .
$$

It is clear that $u$ is Euclidean convex, hence H-convex; its regularity implies that $\partial_{H} u(g)=$ $\left\{\nabla_{H} u(g)\right\}=T(g)$, for every $g=(x, y, t) \in \mathbb{H}$. By Theorem 3.2, $T$ is maximal H-cyclically monotone.

On the other hand, if we consider the matrix $Q=\left(\begin{array}{cc}3 & 0 \\ -2 & 4\end{array}\right)$, the related operator $\tilde{T}$ is again maximal monotone. The map $T$ is defined by

$$
T(x, y, t)=\tilde{T}(x, y)=(3 x,-2 x+4 y) .
$$


Let us prove that $T$ is maximal $\mathrm{H}$-monotone, but not maximal $\mathrm{H}$-cyclically monotone. The maximality follows from the continuity of $T$; besides, the H-monotonicity can be inferred from the monotonicity of $\tilde{T}$. Suppose that $T$ is H-cyclically monotone: then, $\operatorname{dom}(T)=\mathbb{H}$ and Theorem 3.1 imply that there exists an H-convex function $u: \mathbb{H} \rightarrow \mathbb{R}$ such that $\operatorname{gph}(T) \subset \operatorname{gph}\left(\partial_{H} u\right)$; the maximality of $T$ gives that $T=\partial_{H} u$. Since $\partial_{H} u(g)$ is a singleton for all $g \in \mathbb{H}$, then $u$ is Pansu differentiable within $\mathbb{H}$ and $\partial_{H} u(g)=\left\{\nabla_{H} u(g)\right\}$ for every $g \in \mathbb{H}$ (see Theorem 1.3 in [11]). Hence, we have that

$$
\nabla_{H} u(g)=\left(u_{x}+2 y u_{t}, u_{y}-2 x u_{t}\right)=(3 x,-2 x+4 y)=T(g) .
$$

An easy computation shows that a function $u$ satisfying the previous equality does not exist, hence $T$ is not H-cyclically monotone.

\section{The main result}

This section is devoted to the more subtle result, where we prove the horizontal version of the Minty theorem contained in Theorem 1.1.

In order to prove the assertion, we need the following two lemmata:

Lemma 4.1 Let $u: \mathbb{H} \rightarrow \mathbb{R}$ be an $H$-convex function, and $g_{0} \in \mathbb{H}$. Consider the function $f: H_{g_{0}} \rightarrow \mathbb{R}$ defined by

$$
f(g)=u(g)+\frac{\left|\xi_{1}(g)\right|^{2}}{2}
$$

Then, $\lim _{\left|\xi_{1}(g)\right| \rightarrow \infty} f(g)=+\infty$.

Proof: Take $v \in \partial_{H} u\left(g_{0}\right)$; then, for any $g \in H_{g_{0}}$,

$$
u(g) \geq u\left(g_{0}\right)+\left\langle v, \xi_{1}(g)-\xi_{1}\left(g_{0}\right)\right\rangle=\left\langle v, \xi_{1}(g)\right\rangle+u\left(g_{0}\right)-\left\langle v, \xi_{1}\left(g_{0}\right)\right\rangle .
$$

Hence, for $g \in H_{g_{0}}$ and $c=u\left(g_{0}\right)-\left\langle v, \xi_{1}\left(g_{0}\right)\right\rangle$,

$$
f(g) \geq\left\langle v, \xi_{1}(g)\right\rangle+c+\frac{\left|\xi_{1}(g)\right|^{2}}{2},
$$

therefore we get the assertion.

Lemma 4.2 Let $T: \mathbb{H} \rightrightarrows V_{1}$. Let us consider a fixed point $g_{0} \in \mathbb{H}$ and the map $T_{0}(g)=$ $T\left(g_{0} \circ g\right)$. Then,

i. $T$ is H-monotone if and only if $T_{0}$ is H-monotone;

ii. $\left.\operatorname{rge}\left(T_{0}+\xi_{1}\right)\right|_{H_{g}}=V_{1}, \forall g \in \mathbb{H}$, if and only if $\left.\operatorname{rge}\left(T+\xi_{1}\right)\right|_{H_{g}}=V_{1}, \forall g \in \mathbb{H}$.

Proof: i. Let $T$ be H-monotone. Suppose that $v \in T_{0}(g), g^{\prime} \in H_{g}, v^{\prime} \in T_{0}\left(g^{\prime}\right)$; then we have that $v \in T\left(g_{0} \circ g\right), g_{0} \circ g^{\prime} \in H_{g_{0} \circ g}$ and $v^{\prime} \in T\left(g_{0} \circ g\right)$. In addition, $\xi_{1}(g)-\xi_{1}\left(g^{\prime}\right)=$ $\xi_{1}\left(g_{0} \circ g\right)-\xi_{1}\left(g_{0} \circ g^{\prime}\right)$. Therefore,

$$
\left\langle v-v^{\prime}, \xi_{1}(g)-\xi_{1}\left(g^{\prime}\right)\right\rangle \geq 0
$$

from the H-monotonicity of $T$. Hence $T_{0}$ is H-monotone. The converse can be analogously proved. 
ii. Let us remark that

$$
\begin{aligned}
\left.\operatorname{rge}\left(T+\xi_{1}\right)\right|_{H_{g}} & =\left.\operatorname{rge}\left(T\left(g_{0} \circ\left(g_{0}^{-1} \circ \cdot\right)\right)+\xi_{1}(\cdot)\right)\right|_{H_{g}} \\
& =\left.\operatorname{rge}\left(T\left(g_{0} \circ(\cdot)\right)+\xi_{1}(\cdot)+\xi_{1}\left(g_{0}\right)\right)\right|_{H_{g_{0}^{-1} \circ g}} \\
& =\left.\operatorname{rge}\left(T_{0}+\xi_{1}\right)\right|_{H_{g_{0}^{-1} \circ g}}+\xi_{1}\left(g_{0}\right) .
\end{aligned}
$$

The proof of Theorem 1.1 is based on the following comparison lemma for the horizontal normal map (see Theorem 3.1 in [2]), that is quoted below in a simplified version:

Theorem 4.1 Let $\Omega_{0} \subset \mathbb{H}$ be an open and bounded set, and $u, v: \mathbb{H} \rightarrow \mathbb{R}$ be $H$-convex functions. Let $g_{0} \in \Omega_{0}$ such that $u\left(g_{0}\right) \leq v\left(g_{0}\right)$, and $u \geq v$ on $\partial \Omega_{0} \cap H_{g_{0}}$. If $v \in \partial_{H} v\left(g_{0}\right)$ satisfies the inequality

$$
v(g)>v\left(g_{0}\right)+\left\langle v, \xi_{1}(g)-\xi_{1}\left(g_{0}\right)\right\rangle, \quad \forall g \in \partial \Omega_{0} \cap H_{g_{0}},
$$

then $v \in \partial_{H} u\left(\Omega_{0} \cap H_{g_{0}}\right)$.

We are now in a position to prove our main result.

Proof of Theorem 1.1: Let $T: \mathbb{H} \rightrightarrows V_{1}$ be a maximal H-cyclically monotone map with $\operatorname{dom}(T)=\mathbb{H}$. Theorem $3.2 \mathrm{ii}$. gives that there exists a convex function $u: \mathbb{H} \rightarrow \mathbb{R}$ such that $T=\partial_{H} u$.

First of all notice that it is enough to show that, for every $g_{0} \in \mathbb{H}$, there exists $g \in H_{g_{0}}$ such that $0 \in \partial_{H} u(g)+\xi_{1}(g)$. Indeed, suppose that this assertion is proved. Take any $v \in V_{1}$ and consider the function

$$
u_{v}(g)=u(g)-\left\langle v, \xi_{1}(g)\right\rangle .
$$

The function $u_{v}$ is still H-convex, then there exists $g \in H_{g_{0}}$ such that $0 \in \partial_{H} u_{v}(g)+\xi_{1}(g)$. Since $\partial_{H}\left(-\left\langle v, \xi_{1}(\cdot)\right\rangle\right)=\{-v\}$, for every $g \in \mathbb{H}$, we have the equality

$$
\partial_{H} u_{v}=\partial_{H} u-v
$$

therefore, $0 \in \partial_{H} u(g)-v+\xi_{1}(g)$, or, equivalently, $v \in \partial_{H} u(g)+\xi_{1}(g)$.

Let us fix $g_{0} \in \mathbb{H}$ and consider the H-convex function $\phi: \mathbb{H} \rightarrow \mathbb{R}$ defined by

$$
\phi(g)=u(g)+\frac{\left|\xi_{1}(g)\right|^{2}}{2} .
$$

By Lemma 4.1, $\phi(g) \rightarrow+\infty$ whenever $\left|\xi_{1}(g)\right| \rightarrow \infty$ with $g \in H_{g_{0}}$. This implies that, for every $M>0$, there exists $R>0$ such that

$$
\phi(g) \geq \phi\left(g_{0}\right)+M, \quad \forall g \in \partial B\left(g_{0}, R\right) \cap H_{g_{0}},
$$

where $B(g, r)$ is the open Euclidean ball of center $g \in \mathbb{H}$ and radius $r$. Let us denote by $\tau:=\phi\left(g_{0}\right)+M$ and by $V: \mathbb{R}^{3} \rightarrow \mathbb{R}$ the classical Euclidean convex function whose graph in $\mathbb{H} \times \mathbb{R}$ is the upside-down cone such that the vertex is the point $\left(g_{0}, \phi\left(g_{0}\right)\right) \in \mathbb{H} \times \mathbb{R}$, and the basis is $\partial B\left(g_{0}, R\right) \times \tau \subset \mathbb{H} \times \mathbb{R}$. Since $V$ is Euclidean convex, clearly it is also H-convex. In order to apply Theorem 4.1, we observe that

$$
V\left(g_{0}\right)=\phi\left(g_{0}\right) \quad \text { and } \quad \phi(g) \geq \tau=V(g) \text { for every } g \in \partial B\left(g_{0}, R\right) \cap H_{g_{0}} .
$$


Moreover, we have that $p_{0}:=0 \in \partial_{H} V\left(g_{0}\right)$ and

$$
V(g)>V\left(g_{0}\right)+\left\langle p_{0}, \xi_{1}(g)-\xi_{1}\left(g_{0}\right)\right\rangle, \text { for every } g \in \partial B\left(g_{0}, R\right) \cap H_{g_{0}} .
$$

Theorem 4.1 implies that $0 \in \partial_{H} \phi\left(B\left(g_{0}, R\right) \cap H_{g_{0}}\right)$ and we conclude the first part of the proof.

Now, let $T: \mathbb{H} \rightrightarrows V_{1}$ be a set-valued H-monotone map, with domain $\mathbb{H}$, such that, for every $g_{0} \in \mathbb{H}$,

$$
\left.\operatorname{rge}\left(T+\xi_{1}\right)\right|_{H_{g_{0}}}=V_{1} .
$$

We argue by contradiction and we suppose that there exists $g_{0} \in \mathbb{H}$, and $w \notin T\left(g_{0}\right)$ such that, for every $g \in H_{g_{0}}$, and $v \in T(g)$,

$$
\left\langle w-v, \xi_{1}\left(g_{0}\right)-\xi_{1}(g)\right\rangle \geq 0 .
$$

Let us first consider the case $g_{0}=0$. From the assumptions rge $\left.\left(T+\xi_{1}\right)\right|_{H_{0}}=V_{1}$, therefore

$$
w=\tilde{v}+\xi_{1}(\tilde{g}),
$$

for some $\tilde{g} \in H_{0}$ and $\tilde{v} \in T(\tilde{g})$. From (13), taking $g=\tilde{g}$ in (12), we obtain

$$
-\left\langle\xi_{1}(\tilde{g}), \xi_{1}(\tilde{g})\right\rangle \geq 0
$$

i.e., $\xi_{1}(\tilde{g})=0$. Since $\tilde{g} \in H_{0}$, we deduce that $\tilde{g}=0$, and $w=\tilde{v} \in T(0)$, contradicting our assumption on $w$.

Suppose now that $w \notin T\left(g_{0}\right)$, for some $g_{0} \in \mathbb{H}$, but (12) is satisfied for every $g \in H_{g_{0}}$, $v \in T(g)$. Let us consider the set-valued map $T_{0}(\cdot)=T\left(g_{0} \circ \cdot\right)$ as in Lemma 4.2; clearly, $w \notin T_{0}(0)$. Moreover, for every $g^{\prime}=g_{0}{ }^{-1} \circ g \in H_{0}$ and $v \in T_{0}\left(g^{\prime}\right)$, inequality (12) gives

$$
\left\langle w-v, \xi_{1}(0)-\xi_{1}\left(g^{\prime}\right)\right\rangle=\left\langle w-v, \xi_{1}\left(g_{0}\right)-\xi_{1}(g)\right\rangle \geq 0 .
$$

Since, from Lemma 4.2, $T_{0}$ is monotone, from the first part of the proof we argue that we get a contradiction, i.e. $w \in T_{0}(0)$, or, equivalently, $w \in T\left(g_{0}\right)$.

It is an open question for the authors whether, in Theorem 1.1, the assumption of maximal $\mathrm{H}$-cyclic monotonicity of $T$ can be weakened to maximal $\mathrm{H}$-monotonicity, as in the classical case.

\subsection{The open question of the resolvent $J_{\lambda}$.}

In the setting of Minty's theorem, given a maximal monotone set-valued map $T: X \rightrightarrows X$ where $X$ is a Hilbert space, for any $\lambda>0$ one can define a map $J_{\lambda}$, called resolvent of $T$ and given by

$$
J_{\lambda}: X \rightrightarrows X, \quad J_{\lambda}:=(I+\lambda T)^{-1} .
$$

This map turns out to be non-expansive and single-valued from $X$ to $X$ (see, for example, [13]); furthermore, the Yosida approximation $T_{\lambda}=\left(I-J_{\lambda}\right) / \lambda$ is maximal monotone as well. If we consider, in particular, the map $T=\partial \varphi$, where $\varphi: X \rightarrow[-\infty, \infty]$ is a proper, convex and lower semicontinuous function, a well-known result due to Moreau states that the function

$$
\varphi_{\lambda}(x)=\min _{x^{\prime} \in H}\left(\frac{1}{2 \alpha}\left|x^{\prime}-x\right|+\varphi\left(x^{\prime}\right)\right), \quad \lambda>0
$$


is convex and Fréchet differentiable, with gradient $\varphi_{\lambda}^{\prime}=T_{\lambda}$. In addition, $\varphi_{\lambda}(x)$ converges to $\varphi(x)$ as $\lambda \downarrow 0$ for each $x \in H$.

One may wonder whether a similar property is still true in the Heisenberg setting, by considering the candidate most likeable to play the role of $J_{\lambda}$, i.e.,

$$
J_{\lambda}^{H}:=\left(\xi_{1}+\lambda T\right)^{-1}: V_{1} \rightrightarrows \mathbb{H} .
$$

If we assume that $T$ is maximal H-cyclically monotone, by Theorem $1.1 J_{\lambda}^{H}$ is defined on the whole $V_{1}$. In addition, since $\left.\operatorname{rge}\left(\xi_{1}+\lambda T\right)\right|_{H_{g}}=V_{1}$, for every fixed $g \in \mathbb{H}$, one may wonder whether

$$
J_{\lambda}^{H}: V_{1} \rightrightarrows H_{g}
$$

is a single-valued map. Unfortunately this property is not inherited, even if we restrict our attention to the special but still exhaustive case of the map $T=\partial_{H} u$, where $u$ is a realvalued $\mathrm{H}$-convex function defined on the whole $\mathbb{H}$. The main reason relies on the following fact: once we restrict the map $J_{\lambda}^{H}$ to a fixed horizontal plane $H_{g_{0}}$, all the properties of the horizontal subdifferential map $g \mapsto \partial_{H} u(g), g \in H_{g_{0}}$, are lost, since, by definition, they concern the behaviour of the map only at points that can be related two by two via the condition $g^{\prime} \in H_{g}$ or, equivalently, $g \in H_{g^{\prime}}$ (it suffices to look at the notion of H-monotonicity, for instance).

Let us provide an example.

Example 4.1 Let us consider the gauge function $N: \mathbb{H} \rightarrow \mathbb{R}$ defined as

$$
N(x, y, t)=\left(\left(x^{2}+y^{2}\right)^{2}+t^{2}\right)^{1 / 4} .
$$

It is known that this function is H-convex, but it is not Euclidean convex (see [9]). The associated horizontal subgradient map, for every $g=(x, y, z)$, is given by

$$
\partial_{H} N(g)= \begin{cases}\overline{B(0,1)} & (x, y, t)=(0,0,0) \\ \frac{1}{N^{3}(g)}\left(x\left(x^{2}+y^{2}\right)+y t, y\left(x^{2}+y^{2}\right)-x t\right) & (x, y, t) \neq(0,0,0) .\end{cases}
$$

We will show that there exists $g^{\prime \prime} \in \mathbb{H}$, and $g, g^{\prime} \in H_{g^{\prime \prime}}, g \neq g^{\prime}$, such that

$$
\left(\xi_{1}(g)+\partial_{H} u(g)\right) \cap\left(\xi_{1}\left(g^{\prime}\right)+\partial_{H} u\left(g^{\prime}\right)\right) \neq \emptyset .
$$

Set $g^{\prime}=e=(0,0,0)$, and notice that, for every $g \in \mathbb{H}$, with $\xi_{1}(g) \neq(0,0)$, there exists $g^{\prime \prime} \in H_{e}$ such that $g \in H_{g^{\prime \prime}}$. We will prove that

$$
\left(\xi_{1}(g)+\lambda \partial_{H} N(g)\right) \in \lambda \overline{B(0,1)}=\xi_{1}(e)+\lambda \partial_{H} N(e) .
$$

Straightforward computations lead to the following $(g=(x, y, t))$ :

$$
\left|\xi_{1}(g)+\lambda \partial_{H} N(g)\right|^{2}=\left(x^{2}+y^{2}\right)\left(1+\frac{\lambda^{2}}{N^{2}(g)}+\frac{2 \lambda}{N^{3}(g)}\left(x^{2}+y^{2}\right)\right) .
$$

Since $g$ can be arbitrarily chosen, we can take, for instance, $t=1$, and $x^{2}+y^{2} \leq 1$; then, $N(x, y, t) \geq 1$, and

$$
\left(x^{2}+y^{2}\right)\left(1+\frac{\lambda^{2}}{N^{2}(g)}+\frac{2 \lambda}{N^{3}(g)}\left(x^{2}+y^{2}\right)\right) \leq\left(x^{2}+y^{2}\right)(1+\lambda)^{2} .
$$

Then, if we choose $x, y$ such that $x^{2}+y^{2} \leq \lambda^{2} /(1+\lambda)^{2}$, we get the assertion (14). 
As a by-product, the example above brings to light the following fact:

Remark 4.1 There exist H-convex functions $u: \mathbb{H} \rightarrow \mathbb{R}$ such that

$$
\partial_{H} u(g) \cap \partial_{H} u\left(g^{\prime}\right) \neq \emptyset,
$$

for some $g, g^{\prime}$ in the same horizontal plane $H_{g^{\prime \prime}}$.

\section{References}

[1] J.-P. Aubin and H. Frankowska. Set-Valued Analysis. Birkhäuser, 1990.

[2] Z.M. Balogh, A. Calogero, and A. Kristály. Sharp comparison and Aleksandrov-type maximum principles in Heisenberg groups. arXiv:1305.5638, 2013.

[3] Z.M. Balogh and M. Rickly. Regularity of convex functions on Heisenberg groups. Ann. Scuola Norm. Sup. Pisa Cl. Sci, 2:847-868, 2003.

[4] M. Bardi and F. Dragoni. Subdifferential and properties of convex functions with respect to vector fields. J. Convex Anal., 21, 2014.

[5] A. Bonfiglioli, E. Lanconelli, and F. Uguzzoni. Stratified Lie Groups and Potential Theory for their Sub-Laplacians. Springer, 2007.

[6] A. Calogero and R. Pini. Horizontal normal map on the Heisenberg group. J. Nonlinear Convex Anal., 12(2):287-307, 2011.

[7] A. Calogero and R. Pini. c horizontal convexity on Carnot groups. J. Convex Anal., 19:541-567, 2012.

[8] L. Capogna, D. Danielli, S. Pauls, and J.T. Tyson. An Introduction to the Heisenberg Group and the Sub-Riemannian Isoperimetric Problem. Birkhäuser, Basel, 2007.

[9] D. Danielli, N. Garofalo, and D.M. Nhieu. Notions of convexity in Carnot groups. Comm. Anal. Geom., 11:263-341, 2003.

[10] G. Lu, J.J. Manfredi, and B. Stroffolini. Convex functions on the Heisenberg group. Calculus of Variations, 19:1-22, 2004.

[11] V. Magnani and M. Scienza. Characterizations of differentiability for H-convex functions in stratified groups. to appear in Ann. Sc. Norm. Super. Pisa Cl. Sci., 2010.

[12] R.T. Rockafellar. Convex Analysis. Princeton University Press, 1969.

[13] R.T. Rockafellar and R.J-B. Wets. Variational Analysis. Springer, 2004. 